PROCEEDINGS OF THE

AMERICAN MATHEMATICAL SOCIETY

Volume 133, Number 7, Pages 1985-1992

S 0002-9939(05)07867-6

Article electronically published on February 24, 2005

\title{
GLOBAL EXISTENCE \\ FOR A CLASS OF TRIANGULAR PARABOLIC SYSTEMS ON DOMAINS OF ARBITRARY DIMENSION
}

\author{
DUNG LE AND TOAN TRONG NGUYEN
}

(Communicated by David S. Tartakoff)

\begin{abstract}
A class of triangular parabolic systems given on bounded domains of $\mathbb{R}^{n}$ with arbitrary $n$ is investigated. Sufficient conditions on the structure of the systems are found to assure that weak solutions exist globally.
\end{abstract}

\section{INTRODUCTION}

The purpose of this paper is to investigate the global existence problem for a general class of strongly coupled parabolic systems of the type

$$
\begin{aligned}
& u_{t}=\nabla(P(u, v) \nabla u+R(u, v) \nabla v)+F(u, v), \\
& v_{t}=\nabla(Q(u, v) \nabla v)+G(u, v),
\end{aligned}
$$

which is also supplied with the boundary conditions $\frac{\partial u}{\partial \nu}=\frac{\partial v}{\partial \nu}=0$ on the boundary $\partial \Omega$ of a bounded domain $\Omega$ in $\mathbb{R}^{n}$. The initial conditions are described by $u(x, 0)=$ $u_{0}(x)$ and $v(x, 0)=v_{0}(x), x \in \Omega$. Here $u_{0}, v_{0} \in W^{1, p}(\Omega)$ for some $p>n$.

The fundamental theory of strongly coupled systems such as (1.1) was studied in [1]. The concept of $W^{1, p}$ weak solutions and their local existence was formulated there. One of the important issues, the global existence of solutions, was also discussed. It was pointed out that solutions to (1.1) exist globally in time if one has control on their $L^{\infty}$ norms. It is not surprising that many classical methods, which were developed successfully for regular reaction-diffusion systems to obtain a priori estimates of the supremum norms of solutions, failed to handle (1.1). Not much is known for the global solvability for (1.1).

Firstly, invariance principles were used in 4, 9, to study the boundedness of weak solutions for certain strongly coupled systems. Of course, this method required severe restrictions on the initial data of the solutions. $L^{p}$ techniques in [6, 8, 2 ] and the Lyapunov functional approach in [11, 12, 13] were employed to attack this question. However, not only that these authors must assume certain special structure conditions on their systems but their use of Sobolev imbedding inequalities

Received by the editors February 15, 2004.

2000 Mathematics Subject Classification. Primary 35K57; Secondary 35B65.

Key words and phrases. Cross diffusion systems, boundedness, Hölder regularity.

The first author was supported in part by NSF Grant \#DMS0305219, Applied Mathematics Program.

(C)2005 American Mathematical Society Reverts to public domain 28 years from publication 
forced the domain $\Omega$ to be of dimension at most 5 (or even 2). In particular, they considered the following system:

$$
\left\{\begin{array}{l}
\frac{\partial u}{\partial t}=\Delta\left[\left(d_{1}+a_{11} u+a_{12} v\right) u\right]+F(u, v), \\
\frac{\partial v}{\partial t}=\Delta\left[\left(d_{2}+a_{22} v\right) v\right]+G(u, v)
\end{array}\right.
$$

which was proposed by Shigesada, Kawasaki and Teramoto in [10] to study spatial segregation of interacting species. The functions $F, G$ were taken to be of the form

$$
F(u, v)=u\left(b_{1}-c_{11} u-c_{12} v\right), \quad G(u, v)=v\left(b_{2}-c_{21} u-c_{22} v\right), \text { with } b_{i}, c_{i j}>0 .
$$

It is mathematically interesting to know whether global solvability still holds under a more general setting where the dimension of the domain $\Omega$ can be arbitrary. We answer this question in Section 2 Our results essentially improve the aforementioned works. To illustrate this, we apply our results in Section 2 to (1.2). In fact, we allow a more general system than (1.2) by considering the following forms of $P, Q, R$ in (1.1) (notice the presence of $u$ in $Q(u, v)$ below):

$$
\begin{aligned}
& P(u, v)=d_{1}+a_{11} u+a_{12} v, \quad R(u, v)=b_{11} u, \\
& Q(u, v)=d_{2}+a_{21} u+a_{22} v .
\end{aligned}
$$

Since $u, v$ are population densities, only positive solutions are of interest. We then study these solutions in Section 4, and give the proof of the following.

Theorem 1.1. Assume (1.4) and that $d_{i}, a_{i j}>0, b_{11}>0, i, j=1,2$. In addition, suppose that $F(0, v)=G(u, 0)=0$ for all $u, v$, and

$$
\begin{aligned}
& F(u, v) \text { and } G(u, v) \text { are negative if either } u \text { or } v \text { is sufficiently large, } \\
& \qquad a_{11}>a_{21}, a_{22}>a_{12}, \text { and } a_{22} \neq a_{12}+b_{11} .
\end{aligned}
$$

Then weak solutions to (1.1) with nonnegative initial data are classical and exist globally.

In population dynamics terms, (1.6) means that self-diffusion rates are stronger than cross-diffusion ones. Obviously, the reactions $F, G$ given by (1.3) satisfy our assumption (1.5). Note also that the condition $F(0, v)=G(u, 0)=0$ and maximum principles imply that the solutions stay positive if their initial data are nonnegative.

We would like to remark that the $L^{p}$ bootstrapping methods in [6, 8, 2] cannot apply to our case here. Indeed, a crucial ingredient in those techniques is an estimate of $\nabla v$ that will be used in the bootstrapping argument on the equation for $u$. Such an estimate, using standard results for scalar regular parabolic equations (see [5]) for the equation of $v$, is no longer available here. This is because of the presence of $u$, whose regularity is not yet known, in the diffusion term $Q(u, v)$ of the equation for $v$.

Finally, for the sake of simplicity, we consider here systems of two equations with homogeneous Neumann boundary conditions, even if our main results here could apply to those of more equations and suitable other boundary conditions. Moreover, our method can be generalized to treat strongly coupled parabolic systems with full diffusion matrices to obtain not only $L^{\infty}$ bounds for weak solutions but also their Hölder regularity. Results in this direction will be discussed in [7]. 


\section{The GENERAL CASE}

In this section, we study a solution $(u, v)$ of the general system (1.1) and give sufficient conditions for global existence of $(u, v)$. Since (1.1) is a triangular system, thanks to the results of [1], we need only to show that the $L^{\infty}$ norms $u, v$ are bounded. Our first assumption on the coefficients of (1.1) is

(P.1): The functions $P, Q, R, F, G$ are continuous functions in $(u, v)$. Moreover, $P, Q$ are positive for nonnegative $u, v$, and $u_{0}, v_{0}$ are nonnegative on $\Omega$.

Throughout this section, we consider $W^{1, p}$ weak solutions of 1.1). The reader is referred to [1] for the precise definition of these weak solutions and their local existence. Let $(u, v)$ be a weak solution that exists on $\Omega_{T}=\Omega \times(0, T)$ for some $T>0$.

First of all, as a simple consequence of the maximum principles for scalar parabolic equations, one can prove that $u, v$ stay nonnegative.

By multiplying the equation of $v$ in $(1.1)$ with $\left(v-K_{v}\right)+$ and using the assumption on $G$ we easily prove the following.

Lemma 2.1. There exists a constant $K_{v}>0$, which depends on the initial data $v_{0}$, such that $v(x, t) \leq K_{v}$ for all $(x, t) \in \Omega_{T}$.

Consider the following subset of $\mathbb{R}^{2}$ :

$$
\Gamma=\left\{(u, v): u>0, \quad 0<v<K_{v}\right\}
$$

and the following assumptions.

(H.0): There exist a $C^{2}$ function $H(u, v)$ defined on a neighborhood $\Gamma_{0}$ of $\Gamma$, and a constant $K_{0}$ such that $\left(H_{u} F+H_{v} G\right)(H-K)_{+} \leq 0$ for every $(u, v) \in \Gamma_{0}$ and $K \geq K_{0}$.

(H.1): There exists $\lambda_{1}>0$ such that

$$
\begin{gathered}
{\left[H_{u}(P \nabla u+R \nabla v)+H_{v} Q \nabla v\right] \nabla H \geq \lambda_{1}|\nabla H|^{2},} \\
(P \nabla u+R \nabla v) \nabla H_{u}+Q \nabla v \nabla H_{v} \geq 0,
\end{gathered}
$$

for every $(u, v) \in \Gamma_{K}:=\Gamma \cap\{(u, v): H(u, v) \geq K\}, K \geq K_{0}$, with $K_{0}$ being given in (H.0).

(H.2): If $u \rightarrow \infty$ in $\mathbb{R}^{2}$, then $H(u, v) \rightarrow \infty$.

Here, we write $H_{u}=\frac{\partial}{\partial u} H(u, v), H_{u u}=\frac{\partial^{2}}{\partial u \partial u} H(u, v), \nabla H=\nabla_{x} H(\vec{u}(x))$, and so on. Furthermore, $w_{+}$will denote the nonnegative part $\sup \{w, 0\}$ of a function $w$.

Our main result on the boundedness of weak solutions is the following.

Theorem 2.2. The conditions (P.1), (H.0), (H.1) and (H.2) imply that $u, v$ are bounded.

Proof. Firstly, for nonnegative $\eta \in W^{1,2}(\Omega)$, we can test the equations of $u, v$ respectively by $H_{u} \eta$ and $H_{v} \eta$, add the results, and use (2.3) to get

$$
\int_{\Omega} \frac{\partial H}{\partial t} \eta d x+\int_{\Omega}\left(H_{u}(P \nabla u+R \nabla v)+H_{v} Q \nabla v\right) \nabla \eta d x \leq C \int_{\Omega}\left(H_{u} F+H_{v} G\right) \eta d x .
$$

Here, we have used the homogeneous Neumann boundary conditions so that the boundary integrals, which appear in the integration by parts, are all zero. 
We set $H_{0}=\sup _{x \in \Omega} H\left(u_{0}(x), v_{0}(x)\right)$, which is finite because $u_{0}, v_{0}$ are bounded on $\Omega$. Let $K \geq \max \left\{K_{0}, H_{0}\right\}$ and $\eta$ be $(H-K)_{+}$in (2.4). Integrate the result in $t$ and use (H.0), (2.2) to obtain

$$
\left.\int_{\Omega}(H-K)_{+}^{2} d x\right|_{0} ^{t}+\lambda_{1} \int_{0}^{t} \int_{H \geq K}|\nabla H|^{2} d x d s \leq 0 .
$$

Since $(H-K)_{+}=0$ when $t=0\left(\right.$ as $\left.K \geq H_{0}\right)$, the above shows that $(H-K)_{+}=0$ for all $t$. We conclude that $H \leq K$ on $\Omega$. Condition (H.2) basically says that boundedness of $u, v$ comes from that of $H(u, v)$. Thus, $u, v$ are bounded by some constant depending on $K_{0}$ and the initial data $u_{0}, v_{0}$.

\section{The existence of $H$}

We now see that the assumption on the existence of a function $H$, satisfying (H.1), is crucial for our main results in the previous section. Obviously, it is not clear whether this function ever exists. In this section we will find sufficient conditions on the structure of (1.1) such that we can find such $H$.

Clearly, the conditions (2.2), (2.3) are satisfied if the following quadratics (in $U, V \in \mathbb{R}^{n}$ ) are positive definite:

$$
\begin{aligned}
A_{1}:= & (P-\lambda) H_{u}^{2} U^{2}+\left[R H_{u} H_{v}+(Q-\lambda) H_{v}^{2}\right] V^{2} \\
& +\left[R H_{u}^{2}+(Q+P-2 \lambda) H_{u} H_{v}\right] U V \\
A_{2}:= & P H_{u u} U^{2}+\left(R H_{u v}+Q H_{v v}\right) V^{2}+\left[R H_{u u}+(P+Q) H_{u v}\right] U V .
\end{aligned}
$$

$A_{1}$ is positive definite if the coefficients of $U^{2}, V^{2}$ are nonnegative and its discriminant $\Theta_{1}$ is nonpositive. However, a simple calculation shows that

$$
\Theta_{1}=\left(P H_{u} H_{v}-R H_{u}^{2}-Q H_{u} H_{v}\right)^{2}=H_{u}^{2}\left((P-Q) H_{v}-R H_{u}\right)^{2} .
$$

This suggests that we will require $H$ to fulfill $(P-Q) H_{v}=R H_{u}$. In other words, we will consider the following equations:

$$
\begin{gathered}
f(u, v)=(P-Q) / R, \\
H_{u}=f(u, v) H_{v} .
\end{gathered}
$$

Lemma 3.1. Assume that (3.4) holds. There exists $\lambda>0$ such that $A_{1}$ is positive definite.

Proof. By (3.3) and (3.4), the coefficients of $U^{2}, V^{2}$ in $A_{1}$ are respectively $H_{u}^{2}(P-\lambda)$ and $H_{v}^{2}(R f+Q-\lambda)=H_{v}^{2}(P-\lambda)$. They are nonnegative if we choose $\lambda=\inf _{\Gamma} P$.

To verify the positivity of $A_{2}$ in (3.2), we consider its discriminant $\Theta_{2}$. An easy computation shows that

$$
\Theta_{2}:=\left(R H_{u u}+P H_{u v}+Q H_{u v}\right)^{2}-4 P H_{u u}\left(R H_{u v}+Q H_{v v}\right) .
$$

Differentiating $H_{u}=f H_{v}$, we get $H_{u u}=f_{u} H_{v}+f H_{u v}$ and $H_{u v}=f_{v} H_{v}+f H_{v v}$. Substitute these into $\Theta_{2}$ and simplify to obtain

$$
\Theta_{2}:=\alpha_{1} H_{v v}^{2}+\alpha_{2} H_{v v} H_{v}+\alpha_{3} H_{v}^{2} .
$$


Using (3.3), we easily see that $\alpha_{1}=0$. Similarly, we have

$$
\begin{aligned}
& \alpha_{2}= 2\left(R\left(f_{u}+f f_{v}\right)+P f_{v}+Q f_{v}\right)\left(R f^{2}+P f+Q f\right) \\
&-4 P\left[\left(f_{u}+f f_{v}\right)(R f+Q)+R f^{2} f_{v}\right] \\
&= 4\left(-P R f^{2} f_{v}+P^{2} f_{v} f-P Q f_{u}\right) \\
&=-4 P Q\left(f_{u}-f_{v} f\right), \\
& \alpha_{3}=\left(R\left(f_{u}+f f_{v}\right)+P f_{v}+Q f_{v}\right)^{2}-4 P\left(f_{u}+f f_{v}\right) R f_{v} \\
&=\left(f_{u}+f f_{v}\right)^{2} R^{2}+(P+Q)^{2} f_{v}^{2}+2 R f_{v}\left[\left(f_{u}+f f_{v}\right)(Q-P)\right] \\
&=\left(f_{u}+f f_{v}\right)^{2} R^{2}+(P-Q)^{2} f_{v}^{2}+2 R f_{v}\left(f_{u}+f f_{v}\right)(Q-P)+4 P Q f_{v}^{2} \\
&=\left[\left(f_{u}+f f_{v}\right) R+f_{v}(Q-P)\right]^{2}+4 P Q f_{v}^{2} \\
&=R^{2} f_{u}^{2}+4 P Q f_{v}^{2} .
\end{aligned}
$$

Let $g$ be a solution to (3.4) and $G$ be any differentiable function on $\mathbb{R}$. We observe that $H(u, v)=G(g(u, v))$ is also a solution to (3.4). We will make the following main assumptions of this section.

(H.3): Assume that there exists a connected neighborhood $\Gamma_{K}^{0}$ of $\Gamma_{K}$ such that $g$ belongs to $C^{2}\left(\Gamma_{K}^{0}\right)$. Moreover,

$$
g_{v} \neq 0, \text { and } \alpha_{2}=-4 P Q\left(f_{u}-f_{v} f\right) \neq 0, \quad \forall(u, v) \in \Gamma_{K}^{0} .
$$

(H.4): The quantities $g_{v v} / g_{v}^{2}+\alpha_{3} /\left(\alpha_{2} g_{v}\right), \delta_{12} /\left(f \delta_{11}\right)$ and $f \delta_{21} / \delta_{22}$ are bounded on $\Gamma_{K}$. Here, we denote

$$
\delta_{12}=P\left[f^{2} g_{v v}+\left(f_{u}+f f_{v}\right) g_{v}\right], \quad \delta_{21}=P g_{v v}+R f_{v} g_{v},
$$

and $\delta_{11}=\delta_{22}=P f g_{v}^{2}$.

The existence of $H$ is then given by

Theorem 3.2. Assume (H.3), (H.4) and let $H(u, v)=\exp (\mu g(u, v))$. There exists $\mu$ such that (H.1) holds.

Proof. Thanks to Lemma 3.1 and the choice of $g$, we need only to check the positivity of $A_{2}$. We first show that $\Theta_{2}<0$ on $\Gamma_{K}$ for a suitable choice of $\mu$. Let $G(x)=\exp (\mu x)$. As $H_{v}=G^{\prime} g_{v}, H_{v v}=\left(G^{\prime \prime} g_{v}^{2}+G^{\prime} g_{v v}\right)$, and $G^{\prime \prime} / G^{\prime}=\mu$, we have

$$
\Theta_{2}=H_{v v} H_{v} \alpha_{2}+H_{v}^{2} \alpha_{3}=\left(G^{\prime}\right)^{2} g_{v}^{3} \alpha_{2}\left[\mu+\left(\frac{g_{v v}}{g_{v}^{2}}+\frac{\alpha_{3}}{\alpha_{2} g_{v}}\right)\right] .
$$

Thanks to our assumption (3.6) and because $\Gamma_{K}^{0}$ is connected, the coefficient of $\mu$ never vanishes on $\Gamma_{K}$. That is, either $g_{v}^{3} \alpha_{2}<0$ or $g_{v}^{3} \alpha_{2}>0$ on $\Gamma_{K}$. Because $g_{v v} / g_{v}^{2}+\alpha_{3} /\left(\alpha_{2} g_{v}\right)$ is bounded on $\Gamma_{K}$ and $g_{v}, G^{\prime} \neq 0$, the above shows that $\Theta_{2}<0$ on $\Gamma_{K}$ for a suitable choice of $\mu$ with $|\mu|$ being sufficiently large.

Finally, we show that the coefficients of $U^{2}, V^{2}$ in $A_{2}$ are positive. It suffices to show that the following quantities $\delta_{1}=P H_{u u}$ and $\delta_{2}=\left(R H_{u v}+Q H_{v v}\right)$ are strictly positive on $\Gamma_{K}$. A similar calculation as before yields

$$
\begin{gathered}
\delta_{1}=P\left[f^{2} H_{v v}+\left(f_{u} H_{v}+f f_{v} H_{v}\right)\right]=\exp (\mu g) f \delta_{11}\left[\mu \frac{\delta_{12}}{f \delta_{11}}+\mu^{2}\right], \\
\delta_{2}=(R f+Q) H_{v v}+R f_{v} H_{v}=\exp (\mu g) \frac{\delta_{22}}{f}\left[\mu \frac{f \delta_{21}}{\delta_{22}}+\mu^{2}\right],
\end{gathered}
$$


where $\delta_{i j}$ are defined as in (H.4). Since the coefficients of $\mu, \delta_{12} /\left(f \delta_{11}\right)$ and $f \delta_{21} / \delta_{22}$ are bounded on $\Gamma_{K}$, and $f \delta_{11}, \delta_{22} / f$ are positive, we can choose $|\mu|$ large to have that $\delta_{1}, \delta_{2}>0$ on $\Gamma_{K}$.

\section{Proof of Theorem 1.1}

The boundedness of $v$ was proven in Lemma 2.1 so that we will only concern ourselves with the boundedness of $u$ here. We apply Theorem 2.2 to establish Theorem 1.1.

By Lemma 2.1, we can take $\Gamma_{0}$ to be the strip $\left\{(u, v) \mid u>0,0<v<K_{v}\right\}$. We also see that $f$ of $(3.3)$ is given by

$$
f(u, v)=\frac{d+a u-b v}{u},
$$

where

$$
d=\frac{d_{1}-d_{2}}{b_{11}}, \quad a=\frac{a_{11}-a_{21}}{b_{11}}, \quad b=\frac{a_{22}-a_{12}}{b_{11}} .
$$

Our assumption (1.6) simply means $a>0, b \neq 1$ and $b>0$. Moreover, the equation (3.4) can be solved by methods of characteristics (see [3]). In fact, it is elementary to see that the general solution of (3.4) is given by

$$
g(u, v)=L\left(\frac{u^{b}}{d(b-1)+a b u-b(b-1) v}\right),
$$

where $L$ can be any $C^{1}$ function on $\mathbb{R}$.

Since $a^{2} b>0$ and $F(u, v), G(u, v) \leq 0$ if $u$ is large, we can find $K_{1}>0$ such that if $u \geq K_{1}$, then $F(u, v), G(u, v) \leq 0$ and $a\left[d(b-1)+a b u-b(b-1) K_{v}\right]>1$. We define

$$
\Gamma_{1}:=\left\{(u, v) \in \Gamma_{0} \mid u \geq K_{1}\right\}
$$

and

$$
\hat{g}(u, v)=(b-1) \log \left(\frac{u^{b}}{a[d(b-1)+a b u-b(b-1) v]}\right), \quad(u, v) \in \Gamma_{1} .
$$

Put $G_{0}=\sup \left\{\hat{g}(u, v) \mid u=K_{1}, 0<v \leq K_{v}\right\}$. Let $g(u, v)$ be a $C^{1}$ extension of $\hat{g}(u, v)$ on $\Gamma_{0}$ that satisfies $\sup _{\Gamma_{0} \backslash \Gamma_{1}} g(u, v) \leq G_{0}+1$. We then set $G_{1}:=G_{0}+2$. Obviously, we have

$$
g(u, v) \geq G_{1} \Rightarrow(u, v) \in \Gamma_{1} \Rightarrow u \geq K_{1} .
$$

We study the function $g$ on $\Gamma_{1}$. Firstly, we compute and find

$$
\begin{gathered}
g_{v}=\frac{b(b-1)^{2}}{d(b-1)+a b u-b(b-1) v}, \quad g_{v v}=\frac{g_{v}^{2}}{b-1}, \\
f_{u}=\frac{b v-d}{u^{2}}, \quad f_{v}=-\frac{b}{u} .
\end{gathered}
$$

We then prove the following lemmas.

Lemma 4.1. For $(u, v) \in \Gamma_{1}$, we have $g_{v} \alpha_{2}<0$ and $\alpha_{3} /\left(\alpha_{2} g_{v}\right)$ is bounded.

Proof. By (4.4), we have

$$
f_{u}-f f_{v}=\frac{d(b-1)+a b u-b(b-1) v}{u^{2}} \neq 0, \quad \forall(u, v) \in \Gamma_{1} .
$$


Thus, by (4.3), $g_{v} \alpha_{2}=-4 P Q b(b-1)^{2} / u^{2}<0$ on $\Gamma_{1}$. On the other hand, we write

$$
\frac{\alpha_{3}}{\alpha_{2} g_{v}}=\frac{R^{2} f_{u}^{2}}{\alpha_{2} g_{v}}-\frac{f_{v}^{2}}{\left(f_{u}-f f_{v}\right) g_{v}}
$$

which can be simplified to

$$
-\frac{(b v-d)^{2} b_{11}^{2}}{4 b(b-1)^{2} P Q}-\frac{b}{(b-1)^{2}} .
$$

The above quantity is bounded on $\Gamma_{1}$ since $P \geq d_{1}, Q \geq d_{2}$ and $v$ is bounded. The proof of this lemma is complete.

Lemma 4.2. $\delta_{12} /\left(f \delta_{11}\right)$ and $f \delta_{21} / \delta_{22}$ are bounded on $\Gamma_{1}$.

Proof. We have

$$
\frac{\delta_{12}}{f \delta_{11}}=\frac{g_{v v}}{g_{v}^{2}}+\frac{f_{u}+f f_{v}}{f^{2} g_{v}}=\frac{1}{b-1}+\frac{f_{u}+f f_{v}}{f^{2} g_{v}} .
$$

The last fraction is

$$
-\frac{(d(1+b)+a b u-b(b+1) v)(d(b-1)+a b u-b(b-1) v)}{(d+a u-b v)^{2} b(b-1)^{2}},
$$

which is bounded because $v$ is bounded on $\Gamma_{1}$ and the powers of $u$ in the numerator and denominator are equal (so that the fraction is bounded when $u$ is large).

Next, we have

$$
\frac{f \delta_{21}}{\delta_{22}}=\frac{g_{v v}}{g_{v}^{2}}+\frac{R f_{v}}{P g_{v}}=\frac{1}{b-1}-\frac{b_{11}(d(b-1)+a b u-b(b-1) v)}{\left(d_{1}+a_{11} u+a_{12} v\right)(b-1)^{2}} .
$$

The last fraction is bounded on $\Gamma_{1}$ by the same reason as before.

We have shown that the conditions (H.3) and (H.4) are satisfied on the set $\Gamma_{1}$. In particular, because $g_{v} \alpha_{2}<0$, we see that the factor $\mu$ in the proof of Theorem 3.2 can be chosen to be positive and sufficiently large. Fixing such a constant $\mu$, we then define $H(u, v)=\exp (\mu g(u, v))$. Let $K_{0}=\exp \left(\mu G_{1}\right)$. We see that $H(u, v) \geq K_{0} \Rightarrow g(u, v) \geq G_{1}$. Therefore, thanks to (4.2), we have

$$
\Gamma_{K_{0}}=\left\{(u, v) \in \Gamma_{0} \mid H(u, v) \geq K_{0}\right\} \subset \Gamma_{1} .
$$

The definition of $\Gamma_{1}$, Theorem 3.2 and the above lemmas show that (H.0) and (H.1) are verified on $\Gamma_{1}$. By (4.5), they also hold on $\Gamma_{K_{0}}$. It is easy to see that $g(u, v) \sim \log \left(u^{(b-1)^{2}}\right)$ when $u$ is large so that $H(u, v) \sim u^{\mu(b-1)^{2}}$. Since $\mu>0$ and $H(u, v)$ is bounded on $\Gamma_{0} \backslash \Gamma_{1}$ (by $\exp \left(\mu G_{1}\right)$ ) we easily see that $H(u, v) \rightarrow \infty$ iff $u \rightarrow \infty$. Hence (H.2) also holds. Theorem 2.2 asserts that $u$ is bounded. Our proof of Theorem 1.1 is complete.

\section{ACKNOWLEDGEMENT}

The authors would like to thank the anonymous referee for comments that improve the final version of this paper. 


\section{REFERENCES}

1. H. Amann, Dynamic theory of quasilinear parabolic systems-III. global existence. Math. Z. 202 (1989), 219-250. MR 1013086 (90i:35125)

2. Y.S. Choi, R. Lui and Y. Yamada, Existence of global solutions for the Shigesada-KawasakiTeramoto model with strongly-coupled cross diffusion. Discrete Contin. Dyn. Syst. 10 (2004), 719-730. MR2018876

3. L. C. Evans, Partial Differential Equations. AMS Graduate Studies in Math., vol. 19, 1998. MR1625845 (99e:35001)

4. K.H.W. Küfner, Invariant regions for quasilinear reaction-diffusion systems and applications to a two population model. NoDEA, 3(1996), 421-444. MR1418589(97m:35135)

5. O. A. Ladyzenskaja, V. A. Solonnikov, and N. N. Ural'tseva, Linear and Quasilinear Equations of Parabolic Type. AMS Transl. Monographs, vol. 23, 1968. MR0241822 (39:3159b)

6. D. Le, Cross diffusion systems on $n$ spatial dimensional domains. Indiana Univ. Math. J. 51, No.3(2002), 625-643. MR.1911048 (2003b:35090)

7. D. Le, Global existence for a class of strongly coupled parabolic systems. To appear in Annali di Mat. Pura ed Appl.

8. D. Le, L. Nguyen and T. Nguyen, Shigesada-Kawasaki-Teramoto model on higher dimensional domains. Electronic J. Diff. Eqn., No. 72 (2003), 1-12. MR1993780 (2004d:35100)

9. R. Redlinger, Invariant sets for strongly coupled reaction-diffusion systems under general boundary conditions. Arch. Rat. Mech. Anal., 108(1989), 281-291. MR.1012178|(90k:35137)

10. N. Shigesada, K. Kawasaki and E. Teramoto, Spatial segregation of interacting species. J. Theoretical Biology, 79(1979), 83-99. MR0540951 (80e:92038)

11. Seong-A Shim, Uniform Boundedness and Convergence of Solutions to Cross-Diffusion Systems. J. Diff. Eqn., 185, no. 1 (2002), 281-305. MR1935640 (2003f:35171)

12. A. Yagi, Global solution to some quasilinear parabolic system in population dynamics. Nonlinear Analysis T.M.A., 21, no. 8 (1993),531-556. MR.1245865 (94k:35148)

13. A. Yagi, A priori estimates for some quasilinear parabolic system in population dynamics. Kobe J. Math., 14, no. 2 (1997),91-108. MR1612166 (99k:35178)

Department of Applied Mathematics, University of Texas at San Antonio, 6900 North Loop 1604 West, San Antonio, Texas 78249

E-mail address: dle@math.utsa.edu

Department of Applied Mathematics, University of Texas at San Antonio, 6900 North Loop 1604 West, San Antonio, TeXas 78249

E-mail address: toan.nguyen@utsa.edu 